\title{
Tecnología de Información y Comunicación (TIC) y su práctica en la evaluación educativa
}

\section{Information and Communication Technology (ICT) and its Practice in Educational Evaluation}

\author{
Ronald M. Hernández* \\ Universidad Sn Ignacio de Loyola, Lima, Perú \\ ORCID: https://orcid.org/0000-0003-1263-2454 \\ Isaac Sanchez Cáceres \\ Universidad Nacional Federico Villarreal, Lima, Perú \\ ORCID: https://orcid.org/0000-0003-0469-9421 \\ Jesús Roberto Zarate Hermoza \\ Universidad Nacional Federico Villarreal, Lima, Perú \\ ORCID: https://orcid.org/0000-0002-3304-7417 \\ Daniela Medina Coronado \\ Universidad César Vallejo, Lima, Perú \\ ORCID: https://orcid.org/0000-0002-9180-7613 \\ Telmo Pablo Loli Poma \\ Universidad Nacional Santiago Antúnez de Mayolo, Huaraz, Perú \\ ORCID: https://orcid.org/0000-0002-6770-9407 \\ Georgina Raquel Arévalo Gómez \\ Universidad Nacional Mayor de San Marcos, Lima, Perú \\ ORCID: https://orcid.org/0000-0001-7489-894X
}

Recibido 10-03-19 Revisado 12-05-19 Aprobado 12-01-19 En línea 21-05-19

*Correspondencia

Email: rhernandezv@usil.edu.pe
Citar como:

Hernández, R.M., Sanchez, I., Zarate, J., Medina, D., Loli, T., \& Arévalo, G. (2019). Tecnología de Información y Comunicación (TIC) y su práctica en la evaluación educativa. Propósitos y Representaciones, 7(2), 1-10. doi: http://dx.doi.org/10.20511/pyr2019.v7n2.328. 


\section{Resumen}

Son diferentes las formas que han evolucionado las prácticas pedagógicas y la manera de comprender el proceso de enseñanza y aprendizaje. Con el uso del internet las fuentes de información y de evaluación se amplifican, y los agentes educativos dentro de la formación virtual cumplen un rol de importancia, reflexionando sobre un nuevo paradigma de aprendizaje y de construcción del conocimiento. Desde la revisión teórica llevada a cabo en la presente investigación, se exponen aspectos que orientan a una educación virtual y la tendencia a una práctica evaluativa que no se circunscribe al aula, pues las herramientas de intercomunicación se convierten y contribuyen en una nueva práctica pedagógica centrada en el aprendizaje, dando lugar a un rol más activo a los estudiantes.

Palabras clave: Tecnología de Información y Comunicación; Evaluación educativa; Educación; Formación virtual.

\section{Summary}

The ways in which pedagogical practices have evolved and how to understand the teaching and learning process are different. With the use of the Internet, the sources of information and evaluation are amplified, and educational agents within virtual education play an important role, reflecting on a new paradigm of learning and knowledge construction. From the theoretical review carried out in this document, aspects for virtual education and the tendency to an evaluative practice that does not deal exclusively with the classroom are exposed, since the intercommunication tools become and contribute to a new pedagogical practice focused on learning, giving rise to a more active role for students.

Keywords: Information and Communication Technology; Educational Evaluation; Education; Virtual Training.

\section{Introducción}

La llegada de Internet y los constantes avances tecnológicos actuales conllevan una serie de transformaciones que han repercutido en el mundo educativo dando lugar a nuevas formas de comunicarse, interaccionar, relacionarse, trabajar y aprender (Coll, 2013). Es así, que las TIC se han convertido en una herramienta crucial para el futuro de la educación, estas pueden suponer oportunidades importantes para mejorar la educación de los estudiantes y docentes (Tapia, Martinez, \& de la Luz, 2015).

El reconocimiento que ha alcanzado la educación a distancia, durante los últimos años, se ha convertido en una alternativa de respuesta para la formación profesional, respuesta hacia una creciente demanda de la sociedad a las instituciones educativas (Aguilar, 2004). Este nuevo paradigma educativo, como lo denomina Martín y López (2012) ha llevado a indicar que la formación docente se concibe como un proceso de aprendizaje constante, dinámico e inseparable, de esta manera se consolida al trabajo colaborativo como una herramienta emergente en el campo de la formación docente. Tezanos (2008) considera a la tecnología como la expresión del "nuevo paradigma social", que viene a ser equiparable a la de "un nuevo mundo".

La relación del entorno virtual, como un escenario de aprendizaje, y la colaboración presencial buscan desaparecer las barreras espacio temporales, y abre las puertas a nuevas formas de colaboración, comunicación y espacios con diferentes tiempos y ritmos de aprendizaje (Guitert et al., 2005).

Mencionar la formación virtual hace referencia a la utilización de un sistema tecnológico de comunicación masiva y bidireccional que reemplaza a la actividad presencial en el aula, de 
esta forma se realza la utilización de diversos recursos didácticos que propician el aprendizaje autónomo de los estudiantes (García Aretio, 2014; Almenara, Cejudo \& Lozano 2018).

Para Cabero Almenara, Arancibia Muñoz, Valdivia Zamorano, y Aranedas Riveros (2018) existe una actitud positiva, tanto en los docentes como en los estudiantes, para utilizar acciones formativas virtuales, que permitan movilizar el proceso de enseñanza-aprendizaje. A pesar de los diferentes enfoques teóricos en los que se aborda las TIC, su impacto en el proceso de enseñanza y aprendizaje, focaliza al docente como aquel agente clave, mediador de las prácticas educativas y tecnológicas (Rubilar, Alveal, \& Fuentes, 2017).

\section{Práctica evaluativa usando TIC}

Rodríguez (2005) afirma que "la evaluación, es aquel conjunto de procesos sistemáticos de recogida, análisis e interpretación de información válida y fiable, que en comparación con una referencia o criterio nos permita llegar a una decisión que favorezca la mejora del objeto evaluado" (p.43).

Duart y Martínez (2001) indican que una evaluación sea significativa debe cumplir una serie de características a) Sistemática (actividad periódica, previa aprobación de un programa) b) Objetiva (garantía de una evaluación con una diversa existencia de información) c) Participativa (la organización debe considerar una definición de evaluación) y d) Flexible (trata sobre la metodología de evaluación en base a la organización) concluimos que la evaluación no solo abarca aspectos técnicos, sino también aspecto de organización.

El proceso de evaluación está relacionado con la toma decisiones (Mateo, 1990; Pérez Juste \& García Ramos, 1989), y esto se debe a que se intenta enlazar el proceso de evaluación con el proceso de mejora (Rodríguez, 2005). Cuando existe una integración entre el ordenador y la evaluación, el docente plantea la ayuda que supone integrar las herramientas de la web en la evaluación, este modelo supone una evaluación siempre desde un carácter formativo, es decir, como instrumento para el perfeccionamiento didáctico y, en concreto, de cada uno de sus procesos y componentes curriculares (Fernández Pérez,1994).

Para Montesinos (2004) las TIC pueden ampliar la calidad de información, cuyo contexto dependerá de los objetivos planteados en las sesiones y cursos, de esta manera se busca obtener una evaluación no automatizada sino analística, interpretativa y colaborativa, recordemos que la evaluación puede ser sinónimo de intercambio de información. A continuación, se muestra un modelo de posibilidades de integración de la web en el proceso evaluativo, de esta manera se hace mención a las virtudes que tiene el internet para acceder, generar, transmitir conocimiento.

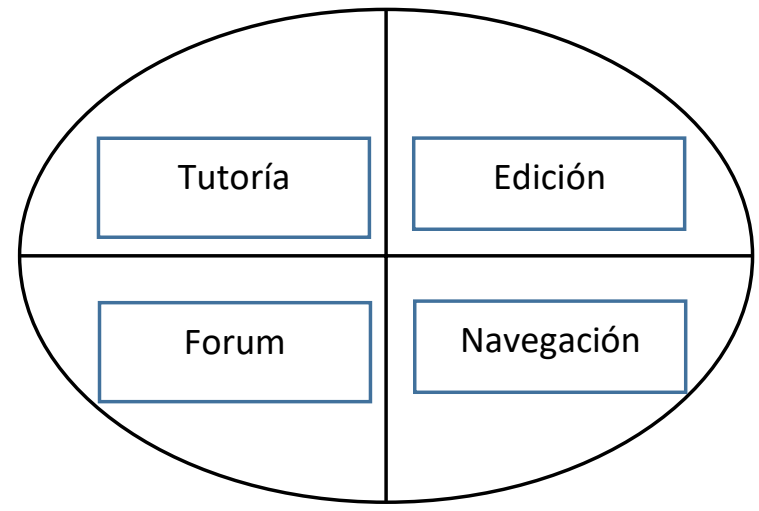

Figura 1. Modelo de posibilidades de integrar Internet en el proceso evaluador (Montesinos 2004). 


\section{Conclusiones}

La formación virtual, basado en las nuevas tecnologías permite favorecer y propiciar los sistemas de evaluación formativa, en base a las autoevaluaciones que permiten al estudiante situarse en el nivel de aprendizaje conseguido y reconducir el proceso hacia niveles superiores de ejecución (Rodriguez, 2005).

Para concluir, es importante mencionar que hoy en día se otorga una valoración exclusiva al planteamiento de la evaluación de programas formativos a través del uso del internet. Se busca entablar acciones formativas para una capacitación en la formación virtual de los docentes, basando su fundamento pedagógico en los aspectos didácticos, referidos al diseño de materiales para la red, o las estrategias y e-actividades que se pueden aplicar en la misma (Cabero-Almenara, Gallego-Pérez, Puentes Puente, \& Jiménez Rosa, 2018).

La evaluación del aprendizaje no solo busca comprender la valoración de los resultados del aprendizaje discente, sino el análisis de los procesos de enseñanza y aprendizaje. Por tanto, la evaluación apoyada con el uso de la tecnología busca: 1) integrar la mejora y comprensión del proceso de aprendizaje, 2) vincular una evaluación formativa y sumativa; 3) destacar que una evaluación usando la web puede ser bidireccional entre docentes y estudiantes; 4) crear la oportunidad de un modelo de evaluación colaborativa y democrática (Montesinos 2004).

\section{Referencias}

Aguilar, R. (2004). La guía didáctica, un material educativo para promover el aprendizaje autónomo. Evaluación y mejoramiento de su calidad en la modalidad abierta ya distancia de la UTPL. RIED. Revista iberoamericana de educación a distancia, 7(1-2), 179-192. https://doi.org/10.5944/ried.7.1-2.1082

Cabero-Almenara, J., Cejudo, M. D. C. L., \& Lozano, J. A. M. (2018). Evaluación del desempeño docente en la formación virtual: ideas para la configuración de un modelo. RIED. Revista Iberoamericana de Educación a Distancia, 21(1), 261-279. Doi: https://doi.org/10.5944/ried.21.1.17206

Cabero-Almenara, J., Arancibia Muñoz, M. L., Valdivia Zamorano, I., \& Aranedas Riveros, S. M. (2018). Percepciones de profesores y estudiantes de la formación virtual y de las herramientas en ellas utilizadas. Revista Diálogo Educacional, 18 (56), 149-163. Doi: http://dx.doi.org/10.7213/1981-416X.18.056.DS07

Cabero-Almenara, J., Gallego-Pérez, O., Puentes Puente, Á., \& Jiménez Rosa, T. (2018). La "Aceptación de la Tecnología de la Formación Virtual" y su relación con la capacitación docente en docencia virtual. EDMETIC, Revista de Educación Mediática y TIC, 7(1), 225-241, Doi: https://doi.org/10.21071/edmetic.v7i1.10028

Duart, J., \& Martínez, M. J. (2001). Evaluación de la calidad docente en entornos virtuales de aprendizaje. Recuperado de: https://cmapspublic2.ihmc.us/rid=1JDJ1SFVP-X2JLXJ$11 \mathrm{MG} /$ Evaluacion_de_la_calidad_docente.pdf

Fernández Pérez, M. (1994). Las tareas de la profesión de enseñar. Madrid, Siglo XXI.

García Aretio, L. (2014). Bases, mediaciones y futuro de la educación a distancia en la sociedad digital. Madrid: Síntesis.

Guitert, M., Lloret, T., Giménez, F., \& Romeu, T. (2005). El treball i l'aprenentatge cooperatius en entorns virtuals: El cas de la Universitat Oberta de Catalunya (UOC). Coneixement $i$

Societat. Revista d'Universitats, Recerca i Societat de la Informació, 8, 44-77. Recuperado de: https://www.raco.cat/index.php/Coneixement/article/view/18373

Martín Sánchez, MA., \& López Meneses, E. (2012). La sociedad de la información y la formación del profesorado. E-actividades y aprendizaje colaborativo. RIED. Revista Iberoamericana de Educación a Distancia, 15(1), 15-35. Recuperado de: http://revistas.uned.es/index.php/ried/article/view/775

Mateo, J. (1990). La toma de decisiones en el contexto de la evaluación educativa. Revista de 
Investigación Educativa, 8, 16, 95-112.

Montesinos, M. T. F. (2004). Evaluar a través de Internet. Píxel-Bit. Revista de Medios y Educación, 24, 79-88. Recuperado de: https://recyt.fecyt.es/index.php/pixel/article/view/61233

Pérez Juste, R., \& García Ramos, J.M. (1989). Diagnóstico, evaluación y toma de decisiones. Madrid: Rialp

Rodríguez, M. (2005). Aplicación de las TIC a la evaluación de alumnos universitarios. Revista Electrónica Teoría de la Educación, 6 (2). Recuperado de: http://revistas.usal.es/index.php/eks/article/view/18185

Rubilar, P. S., Alveal, F. R., \& Fuentes, A. C. M. (2017). Evaluación de la alfabetización digital y pedagógica en TIC, a partir de las opiniones de estudiantes en Formación Inicial Docente. Educação e Pesquisa, 43(1), 127-143. Doi: http://dx.doi.org/10.1590/s15179702201701154907

Tapia, J. A. R., Ávila, M. M., \& Paz, M. D. L. L. S. (2016). El impacto de las TICs en la calidad de la educación superior. Revista de investigación en ciencias contables y administrativas, $\quad$ 1(1), 28-44. Recuperado de: http://ricca.umich.mx/index.php/ricca/article/view/1/45 